\title{
A feasibility study of outpatient chemotherapy with S-1 + cisplatin in patients with advanced gastric cancer
}

\author{
Shunsuke Okazaki $\cdot$ Takako E. Nakajima $\cdot$ Jun Hashimoto $\cdot$ \\ Seiichiro Yamamoto · Daisuke Takahari $\cdot$ Ken Kato $\cdot$ Tetsuya Hamaguchi · \\ Yasuhide Yamada $\cdot$ Yasuhiro Shimada $\cdot$ Kenji Tamura
}

Received: 31 August 2011 / Accepted: 4 January 2012/Published online: 8 February 2012

(c) The International Gastric Cancer Association and The Japanese Gastric Cancer Association 2012

\begin{abstract}
Background Regimens of standard-dose cisplatin have usually been administered as inpatient chemotherapy in Japan. This prospective study evaluated the feasibility of outpatient chemotherapy with standard-dose cisplatin in Japanese patients with advanced gastric cancer.

Methods Advanced gastric cancer patients received an S-1 + cisplatin regimen (S-1: 80-120 mg days 1-21; cisplatin: $60 \mathrm{mg} / \mathrm{m}^{2}$ day 8 , every $4-5$ weeks), either as outpatient chemotherapy with oral hydration on days 9-10, or as inpatient chemotherapy with intravenous hydration on days $9-10$, based on the results of an oral hydration test
\end{abstract}

S. Okazaki · T. E. Nakajima - J. Hashimoto - K. Kato ·

T. Hamaguchi · Y. Yamada · Y. Shimada

Gastrointestinal Oncology Division, National Cancer Center

Hospital, Tokyo, Japan

S. Okazaki

Department of Medical Oncology, National Hospital

Organization Kyoto Medical Center, Kyoto, Japan

T. E. Nakajima $\cdot$ K. Tamura

Outpatient Chemotherapy Center, National Cancer Center

Hospital, Tokyo, Japan

T. E. Nakajima ( $\square)$

Department of Clinical Oncology, St. Marianna University,

School of Medicine, 2-16-1 Sugao, Miyamae-ku,

Kawasaki 216-8511, Japan

e-mail: tnakajima@marianna-u.ac.jp

S. Yamamoto

Statistics and Cancer Control Division, National Cancer Center,

Tokyo, Japan

D. Takahari

Department of Clinical Oncology, Aichi Cancer Center Hospital,

Nagoya, Japan during days 1-7 of the first cycle. The primary endpoint was the completion rate of two cycles in the outpatient group.

Results A total of 36 patients were enrolled: 32 were allocated to the outpatient group and 4 to the inpatient group. The completion rate of two cycles in the outpatient group was 78\% [90\% confidence interval (CI): 63-89]. The median of the total number of treatment cycles of $\mathrm{S}-1+$ cisplatin and the median progression-free survival in the outpatient group were 5 (range 1-11) and 10.6 months (95\% CI 4.2-16.9), respectively. Although seven patients in the outpatient group discontinued treatment, mainly owing to gastrointestinal toxicity, most of them could continue S-1 + cisplatin by switching to inpatient chemotherapy from the next cycle.

Conclusion Outpatient chemotherapy with S-1 + cisplatin in advanced gastric cancer patients can be safely and effectively administered in Japan with appropriate patient selection and supportive treatment.

Keywords Cisplatin - Gastric cancer - Oral hydration · Outpatient chemotherapy $\cdot$ S-1

\section{Background}

Gastric cancer is the most common cause of morbidity from cancer in Japan, and despite a decrease in recent years its mortality rate is still as high as $16 \%$ among all cancers [1]. Unresectable or recurrent gastric cancer has an extremely poor prognosis, so the establishment of a standard chemotherapy regimen is a high priority. Since the FAMTX [5-fluorouracil (5-FU) + doxorubicin + methotrexate] regimen demonstrated a survival advantage over best 
supportive care [2], various regimens have been developed around the world.

Although a 5-FU continuous infusion regimen had long been considered the standard in Japan for unresectable or recurrent gastric cancer, based on the results of the Japan Clinical Oncology Group (JCOG) 9205 trial [3], an S-1 (tegafur, 5-chloro-2,4-dihydropyrimidine, and potassium oxonate) + cisplatin (CDDP) regimen recently replaced it, on the basis of the JCOG 9912 trial [4] and SPIRITS trial [5], and this regimen is now widely used. In China, as well as in Japan, S-1 + CDDP is regarded as one of the standard treatments for unresectable or recurrent gastric cancer, based on the results of a domestic randomized controlled trial [6]. The FLAGS trial was conducted in Western countries to prove the superiority of S-1 + CDDP over $5-\mathrm{FU}+\mathrm{CDDP}$, and S-1 + CDDP demonstrated better overall survival (although without statistical significance) and a significantly better safety profile [7].

Outpatient chemotherapy has gradually become popular in Japan with the spread of outpatient chemotherapy units [8]. However, regimens containing standard-dose CDDP $\left(\geq 50 \mathrm{mg} / \mathrm{m}^{2}\right)$ have usually been administered as inpatient chemotherapy because the regimen is highly emetic and requires intensive hydration [9], although it is commonly administered as outpatient chemotherapy in Western countries. According to a recent conference presentation in Japan, only $3.7 \%$ of chemotherapy with standard-dose CDDP was administered on an outpatient basis in Japan. The drug lag of new-generation antiemetics, plus delays in the development of antiemetic guidelines and in the improvement of the infrastructure of outpatient chemotherapy units, are other reasons for the high proportion of inpatient treatment. This situation decreases the quality of life of patients and imposes a large financial burden due to the hospitalization cost.

In recent years, some encouraging reports have emerged from Japan. A report on the safety data of a retrospective study in lung cancer patients who received standard-dose CDDP with a "short hydration regimen [10]", which was already common in Western countries, was presented at a recent meeting in Japan. However, it is assumed that oral hydration is unsuitable for advanced gastric cancer patients, due to their specific conditions, such as the stenosis caused by the primary lesion, anorexia in postgastrectomy patients, and potential bowel obstruction due to peritoneal dissemination. We performed a single-institute retrospective study in 45 advanced gastric cancer patients receiving $\mathrm{S}-1+\mathrm{CDDP}$ as the first-line treatment, and found that 15 patients $(33 \%)$ with gastrointestinal toxicity of grade 1 or less for their entire treatment period could be potential candidates for safely receiving standard-dose CDDP as outpatient chemotherapy. This paper reports on a prospective study conducted to evaluate the feasibility of outpatient chemotherapy with S-1 + CDDP in advanced gastric cancer patients in Japan.

\section{Patients and methods}

\section{Patients}

Eligibility criteria were as follows: unresectable or recurrent gastric cancer, or advanced gastric cancer which required neoadjuvant chemotherapy with S-1 + CDDP; histologically confirmed gastric adenocarcinoma; an Eastern Cooperative Oncology Group (ECOG) performance status of 0-2; aged between 20 and 75 years; agreed to take oral hydration of $\geq 1.5 \mathrm{~L} /$ day; and adequate organ functions: neutrophil count $\geq 1200 / \mathrm{mm}^{3}$, hemoglobin level $\geq 8.0 \mathrm{~g} / \mathrm{dL}$, platelet count $\geq 75000 / \mathrm{mm}^{3}$, total bilirubin level $\leq 3.0 \mathrm{mg} / \mathrm{dL}$, and aspartate aminotransferase and alanine aminotransferase levels $\leq 100 \mathrm{IU} / \mathrm{L}$. In terms of renal function, either a serum creatinine level of $\leq 1.5 \mathrm{mg} / \mathrm{dL}$ or an estimated creatinine clearance of $\geq 50 \mathrm{~mL} / \mathrm{min}$ was required. Prior chemotherapy was allowed except for a CDDP-containing regimen, and measurable lesions were not mandatory. Although the conditions of patients with and without prior chemotherapy might have been different, we considered it allowable to include patients with prior chemotherapy (except for those who had received CDDP previously), because this study did not actually aim to prove the efficacy and safety of S-1 + CDDP.

Written informed consent was obtained from all patients, and the study was approved by the institutional review boards of each of the two institutions involved. The study was monitored for the entire time by an independent data and safety monitoring committee, and kept within the Helsinki Declaration and Japanese Ethical Guidelines for Clinical Studies. This study is registered with University hospital Medical Information Network Clinical Trials Registry (UMIN-CTR), number 000002685.

\section{Treatments}

The study schema is shown in Fig. 1. S-1 was administered orally twice daily at a dose of $80-120 \mathrm{mg} /$ day [80 mg/day for a body surface area (BSA) of $<1.25 \mathrm{~m}^{2}, 100 \mathrm{mg} /$ day for a BSA of 1.25-1.5 $\mathrm{m}^{2}$, and $120 \mathrm{mg} /$ day for a BSA of $\geq 1.5 \mathrm{~m}^{2}$ ] for 21 consecutive days, followed by a 14-day rest; and CDDP was administered intravenously at a dose of $60 \mathrm{mg} / \mathrm{m}^{2}$ on day 8 . The protocol treatment was defined as two cycles, and we recommended continuing S-1 + CDDP as a subsequent treatment when it was effective. When administered as neoadjuvant chemotherapy, the rest period could be shortened to a minimum of 7 days. Patients were given an oral hydration test during 


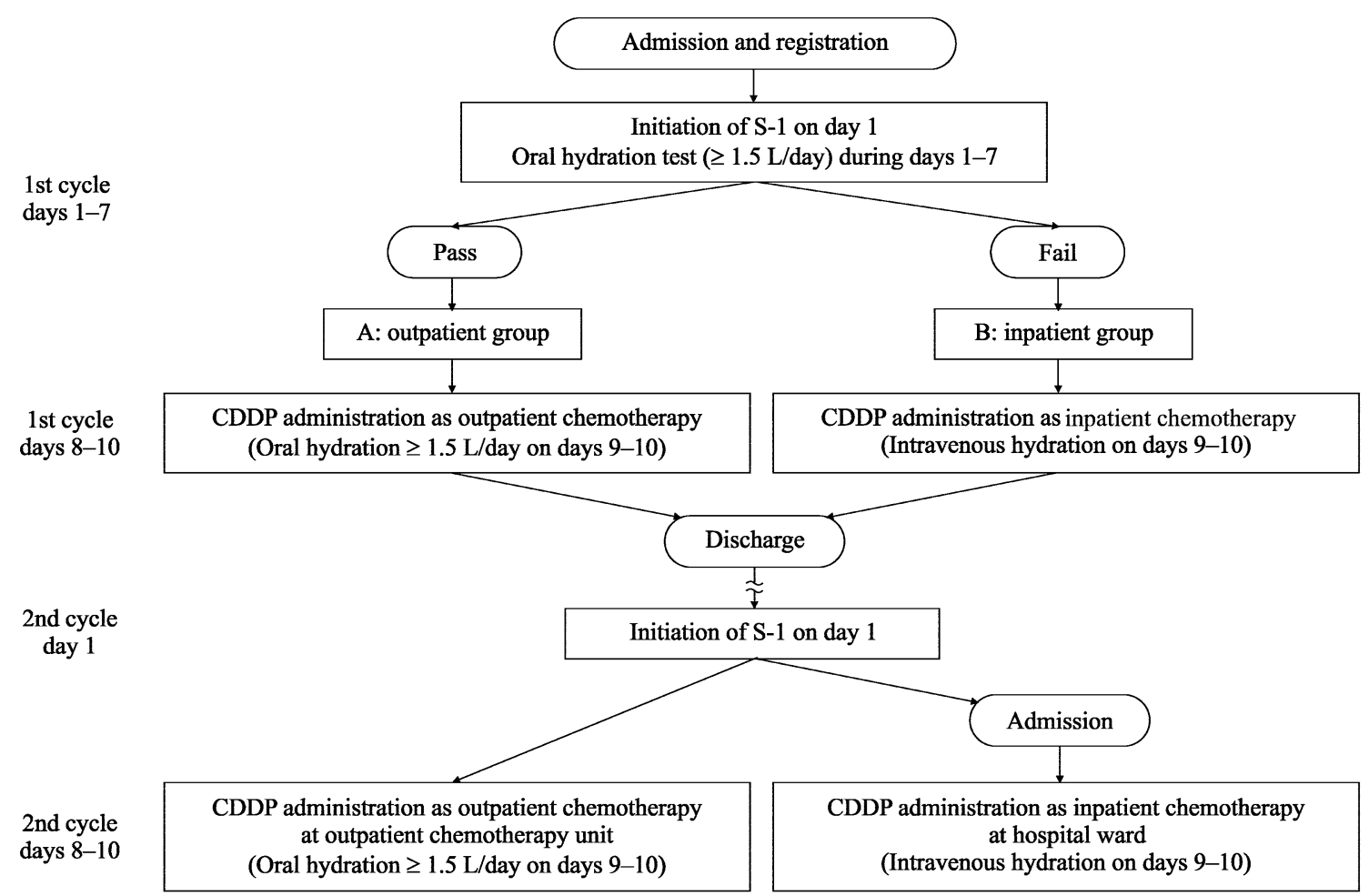

Fig. 1 Flow chart of protocol treatment. $C D D P$ cisplatin

days 1-7 of the first cycle. In the oral hydration test, patients were required to take oral hydration of $\geq 1.5 \mathrm{~L} /$ day in addition to food every day throughout days 1-7. Patients wrote down the self-reported amount of oral hydration every day throughout days 1-7, and the physicians checked it by the next morning. Chemotherapy in the first cycle was initiated after hospital admission in every patient, to enable an accurate check of the amount of oral hydration. Patients were allocated to the outpatient group (group A) if they passed the oral hydration test (or if they could take oral hydration of $\geq 1.5 \mathrm{~L} /$ day every day throughout days $1-7$ ), and they were allocated to the inpatient group (group B) if they failed the test. Group A patients were administered intravenous hydration only on day 8 , and were encouraged to take oral hydration of $\geq 1.5 \mathrm{~L} /$ day on days $9-10$. They were then discharged, and continued to take S-1 until day 21. In the second cycle they were not given an oral hydration test, and CDDP was administered at the outpatient chemotherapy unit on day 8. Group B patients were administered intravenous hydration of the same amount as group A patients on day 8 and 2 L/day on days 9-10, and CDDP was administered in the hospital ward on day 8 in the second cycle.

The CDDP administration method in group A is shown in Table 1. In group A, a total of $2300 \mathrm{~mL}$ hydration was administered in $5 \mathrm{~h}$ and $15 \mathrm{~min}$. Patients were orally administered antiemetics on days 9-11 and diuretics when
Table 1 Cisplatin administration method in group A

\begin{tabular}{llll}
\hline Day 8 & & & \\
\hline Normal saline & $1000 \mathrm{~mL}$ & Drip infusion & 2 hours \\
\hline $\begin{array}{l}\text { Dexamethasone } \\
\text { Granisetron } \\
\text { Normal saline }\end{array}$ & $\begin{array}{l}20 \mathrm{mg} \\
1 \mathrm{mg}\end{array}$ & Drip infusion & 15 minutes \\
\hline $\begin{array}{l}\text { Cisplatin } \\
\text { Normal saline }\end{array}$ & $60 \mathrm{mg} / \mathrm{m}^{2}$ & Drip infusion & 1 hour \\
\hline Normal saline & $1000 \mathrm{~mL}$ & Drip infusion & 2 hours \\
\hline Days 9-10 & & & \\
\hline Oral hydration & $\geq 1.5 \mathrm{~L}$ & Orally & \\
\hline Days 9-11 & & & \\
\hline $\begin{array}{l}\text { Dexamethasone } \\
\text { Granisetron }\end{array}$ & $16 \mathrm{mg}$ & Orally \\
\hline Furosemide & $40 \mathrm{mg}$ & Orally (as needed) \\
\hline
\end{tabular}

they gained weight by $\geq 1.5 \mathrm{~kg}$ in relation to their weight on day 8. Group B patients were administered these drugs intravenously. The regimen of antiemetics was in accordance with the 1999 American Society of Clinical Oncology (ASCO) guidelines [11], because newer agents such as aprepitant and palonosetron had not been approved in Japan when the study was being planned. Group A patients were required to contact their physicians immediately when they could not take adequate oral hydration on days 
9-10, and they were administered intravenous hydration as needed, resulting in discontinuation of the protocol treatment. Even in these patients, S-1 + CDDP could be continued. The completion of treatment was defined as follows: had taken $S-1$ for $\geq 14$ days per cycle, had been administered CDDP, and had not met the discontinuation criteria for S-1 + CDDP. Treatment discontinuation was required in any of the following cases: inadequate oral hydration despite a lack of major toxicity or intravenous hydration on days 9-10 (group A only), administration of second-cycle CDDP as inpatient chemotherapy at the physician's discretion (group A only), disease progression, unacceptable toxicity, patient's refusal, and physician's judgment.

Physical and blood examinations were required every week in the first cycle and every other week in the second cycle. The toxicity was evaluated according to the Common Terminology Criteria for Adverse Events (CTCAE), version 3.0. Imaging tests were performed after the completion of two cycles, and the response was evaluated with the Response Evaluation Criteria in Solid Tumors (RECIST) version 1.0 in those patients with measurable lesions.

\section{Statistical analysis}

The completion rates of two cycles in the SPIRITS trial and in our retrospective study were $70 \%$ (90\% CI 63-76) and $78 \%$ (90\% CI 65-87), respectively, as the first-line treatment in the hospital ward. Because the patients were recruited to this study regardless of their history of prior chemotherapy (except for those who had received CDDP previously), and CDDP was administered as outpatient chemotherapy in group A, a 50\% completion rate of two cycles in group A was considered acceptable. Given that the point estimate was around $50 \%$, and the $90 \%$ CI was between 30 and 70\%, 20 patients were required in group A. Therefore, given that the proportion allocated to group A was around $60 \%$, and in expectation of a few ineligible patients, the total sample size required was determined to be 35 patients.

The primary endpoint was the completion rate of two cycles in group A. The secondary endpoints were as follows: the completion rate of two cycles in group B, progression-free survival in each group, and adverse events in each group. Progression-free survival was defined as the period from the registration date to the earlier of the first radiological or clinical disease progression or death from any cause. In the event that neither disease progression nor death occurred, the case was censored at the last date confirmed as progression-free. When a patient in the neoadjuvant setting could subsequently receive curative surgery, the case was censored at the date of surgery.
A follow-up survey was scheduled 1 year from the date of the last registration. Progression-free survival was analyzed using the Kaplan-Meier method. All of the analyses were performed by an intention-to-treat method, using SAS version 9.2 .

\section{Results}

A total of 36 patients were registered from two institutions in Japan between October 2008 and July 2009; 32 patients were allocated to group A and 4 to group B. Patient characteristics in each group are shown in Table 2. In terms of ECOG performance status, all the patients were either 0 or 1 . In five patients the regimens were administered as neoadjuvant chemotherapy, and all five of these patients were allocated to group A. Seven patients (22\%) in group A and one $(25 \%)$ in group B had a history of prior chemotherapy (Table 3 ). The median follow-up period was 13.0 months (range 3.0-21.3).

The number of patients who completed the protocol treatment was 25 in group A, and 4 in group B. Therefore, the completion rate of two cycles was $78 \%$ (90\% CI 63-89) in group A, and 100\% (90\% CI 47-100) in group B. Of the five patients who received S-1 + CDDP as neoadjuvant chemotherapy, two could receive curative surgery subsequently. Of the seven patients $(22 \%)$ in group A who could not complete the protocol treatment, six could continue

Table 2 Patient characteristics

\begin{tabular}{lll}
\hline & $\begin{array}{l}\text { Group A } \\
N=32\end{array}$ & $\begin{array}{l}\text { Group B } \\
N=4\end{array}$ \\
\hline $\begin{array}{ll}\text { Median age, years (range) } \\
\text { Sex }\end{array}$ & $62(34-75)$ & 65 (59-75) \\
Male & 22 & 4 \\
Female & 10 & 0 \\
ECOG performance status & & \\
0 & 13 & 1 \\
1 & 19 & 3 \\
Treatment line & & \\
Neoadjuvant chemotherapy & 5 & 3 \\
First line & 20 & 1 \\
Second line & 4 & 0 \\
Third line & 3 & 2 \\
Primary lesion & & 2 \\
Yes & 27 & 3 \\
No & 5 & 1 \\
Peritoneal metastasis & & \\
Yes & 20 & \\
No & 12 & \\
\hline
\end{tabular}

ECOG Eastern Cooperative Oncology Group 
S-1 + CDDP as inpatient chemotherapy from the next cycle, whereas one was forced to switch to S-1 monotherapy due to grade 3 anorexia, nausea, and diarrhea.

Adverse events in each group are shown in Table 4. In regard to the elevation of the serum creatinine level in group A, creatinine of all-grade and grade 3-4 was found in 14 patients $(44 \%)$ and none $(0 \%)$, respectively. All-grade creatinine promptly resolved in most patients: it was still found only in 5 patients $(16 \%)$ at the completion of the second cycle, and in the same number at the completion of the S-1 + CDDP regimen. There were no treatment-related deaths or early deaths within 30 days after treatment discontinuation.

In group A, delay in initiation of the second cycle due to adverse events occurred in six patients (19\%), dose reduction related to adverse events was required in five patients $(16 \%)$, and treatment discontinuation was required in seven patients $(22 \%)$ for the following reasons: six had severe gastrointestinal toxicity, and one had inadequate

Table 3 Prior chemotherapy

\begin{tabular}{lll}
\hline & $\begin{array}{l}\text { Group A } \\
N=32\end{array}$ & $\begin{array}{l}\text { Group B } \\
N=4\end{array}$ \\
\hline First line & & \\
S-1 monotherapy & 5 & 1 \\
5-FU continuous infusion & 1 & 0 \\
5-FU + methotrexate & 1 & 0 \\
Second line & & \\
NK105 (micelle product of paclitaxel) & $3^{\text {a }}$ & 0 \\
\hline 5-FU 5-fluorouracil & & \\
a All patients received S-1 monotherapy as first line &
\end{tabular}

oral hydration on days $9-10$ despite a lack of major toxicity.

The median of the total number of treatment cycles of S-1 + CDDP, including subsequent treatment after the third cycle, was 5 (range 1-11) in group A, and 5 (range 2-7) in group B. Median progression-free survivals in group A and B were 10.6 months (95\% CI 4.2-16.9) and 5.6 months (95\% CI 1.7-9.5), respectively (Fig. 2).

\section{Discussion}

In the present study, the completion rate of two cycles in group A was 78\% (90\% CI 63-89), and this met the predefined threshold. In addition, all the other endpoints, as well as the median of the total number of treatment cycles of S-1 + CDDP, were equal to or better than those found in previous reports $[5,12]$.

Based on these positive results in both groups, we conclude that outpatient chemotherapy with standard-dose CDDP in advanced gastric cancer patients is feasible, and that an oral hydration test has the potential to be appropriate for patient selection into outpatient chemotherapy or inpatient chemotherapy. To our knowledge, this is the first prospective study in Japan to have proven that outpatient chemotherapy with standard-dose CDDP can be performed safely.

The major reason for treatment discontinuation in the seven patients in group A was severe gastrointestinal toxicity. We could not find major differences in patient characteristics between those who could and those who could

Table 4 Adverse events

\begin{tabular}{|c|c|c|c|c|c|}
\hline \multirow[t]{2}{*}{ Table 4 Adverse events } & \multirow[t]{2}{*}{ CTCAE v 3.0} & \multicolumn{2}{|l|}{$\begin{array}{l}\text { Group A } \\
N=32\end{array}$} & \multicolumn{2}{|l|}{$\begin{array}{l}\text { Group B } \\
N=4\end{array}$} \\
\hline & & $\begin{array}{l}\text { All grades } \\
N(\%)\end{array}$ & $\begin{array}{l}\text { Grades 3-4 } \\
N(\%)\end{array}$ & $\begin{array}{l}\text { All grades } \\
N(\%)\end{array}$ & $\begin{array}{l}\text { Grades 3-4 } \\
N(\%)\end{array}$ \\
\hline & Leukocytes & $23(72)$ & $3(9)$ & $3(75)$ & $0(0)$ \\
\hline & Neutrophils & $14(44)$ & $7(22)$ & $3(75)$ & $0(0)$ \\
\hline & Hemoglobin & $32(100)$ & $2(6)$ & $3(75)$ & $0(0)$ \\
\hline & Platelets & $13(41)$ & $0(0)$ & $2(50)$ & $0(0)$ \\
\hline & Febrile neutropenia & $1(3)$ & $0(0)$ & $0(0)$ & $0(0)$ \\
\hline & Infection with normal ANC & $2(6)$ & $1(3)$ & $0(0)$ & $0(0)$ \\
\hline & Creatinine & $14(44)$ & $0(0)$ & $0(0)$ & $0(0)$ \\
\hline & Fatigue & $20(63)$ & $0(0)$ & $3(75)$ & $0(0)$ \\
\hline & Anorexia & $27(84)$ & $4(13)$ & $4(100)$ & $0(0)$ \\
\hline & Nausea & $22(69)$ & $2(6)$ & $3(75)$ & $0(0)$ \\
\hline & Vomiting & $7(22)$ & $1(3)$ & $1(25)$ & $0(0)$ \\
\hline \multirow{3}{*}{$\begin{array}{l}\text { CTCAE Common Terminology } \\
\text { Criteria for Adverse Events, } \\
v \text { version, } A N C \text { absolute } \\
\text { neutrophil count }\end{array}$} & Stomatitis & $17(53)$ & $0(0)$ & $2(50)$ & $0(0)$ \\
\hline & Diarrhea & $17(53)$ & $3(9)$ & $2(50)$ & $0(0)$ \\
\hline & Constipation & $15(47)$ & $0(0)$ & $2(50)$ & $0(0)$ \\
\hline
\end{tabular}




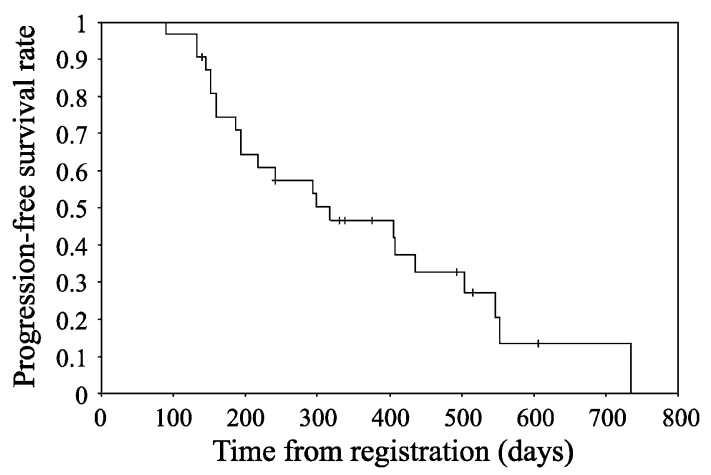

Fig. 2 Progression-free survival in group A

not complete the protocol treatment, and it is difficult to predict such toxicities in advance. Six out of these seven patients in group A were able to continue S-1 + CDDP by switching to inpatient chemotherapy from the next cycle. In addition, another two patients who completed the protocol treatment could also continue it by switching to inpatient chemotherapy in their subsequent treatment. Of the 31 patients in group A who received not less than two cycles of S-1 + CDDP, 23 patients stayed in group A for the entire time and 8 moved to group $B$ subsequently. The proportion of any toxicity of grade 3 or more was $13 \%$ in patients who stayed in group A, and $38 \%$ in patients who moved to group $\mathrm{B}$, indicating a certain level of difference; however, almost all the patients who moved to group $B$ experienced these severe toxicities during the treatments in group $\mathrm{A}$, not after they moved to group B. In addition, all-grade creatinine (39 and 25\%), median progression-free survival (7.0 and 8.0 months), and the median of the total number of treatment cycles of S-1 + CDDP (4 and 5) were all comparable in these two subgroups: patients who stayed in group A and those who moved to group B. This study therefore also proved that S-1 + CDDP can be safely continued even if it is initiated as outpatient chemotherapy and subsequently has to be switched to inpatient chemotherapy.

The present study has several limitations. Firstly, we prospectively examined only two cycles as the protocol treatment. This is because we included patients in the neoadjuvant setting in the target population, and we usually give such patients no more than two cycles of S-1 + CDDP. However, the median of the total number of treatment cycles of S-1 + CDDP was equal to or better than that found in previous reports [5, 12], so we do not consider this to be a major issue.

Secondly, there is insufficient evidence for the adequacy of the specified volume, $1.5 \mathrm{~L} /$ day, of oral hydration we used, both during the period of the oral hydration test and on days 9-10. To our knowledge, there has been no randomized controlled trial to determine an adequate infusion volume on the day of CDDP administration, and there is far less evidence for the amount of oral hydration required before or after the day of CDDP administration. A report on the pharmacokinetics of CDDP showed that the blood level of free CDDP, which causes renal toxicity, diminished below the limit of detection sensitivity on the day after CDDP administration [13], and it is thought that hydration on the day after CDDP administration may not be indispensable.

Thirdly, the regimen of antiemetics we used was outdated because of the drug-lag of approval of new-generation antiemetics in Japan. Aprepitant was finally approved in Japan in October 2009 and palonosetron was approved in January of 2010.

Outpatient chemotherapy with standard-dose CDDP in Japan can also be justified from a cost standpoint. According to the estimate at our institution, the treatment cost per cycle was JPY 131,834 in group A (fee-for-service and drug cost for three visits) and JPY 317,860 in group B (fee-for-service for two visits and Diagnosis Procedure Combination cost for 6-day hospitalization). The cost of outpatient chemotherapy administration in group A was therefore as low as $42 \%$ of that of the inpatient administration in group B.

This finding does not mean we ought to force outpatient chemotherapy on those who do not want to accept oral hydration, or those who strongly desire to receive inpatient chemotherapy. However, we should keep in mind that many more patients prefer outpatient chemotherapy to inpatient chemotherapy $[14,15]$, even when the regimen contains standard to highdose CDDP [16]. Also, it may be possible to give outpatient chemotherapy from the first cycle without an oral hydration test for patients who agree to take oral hydration of $\geq 1.5 \mathrm{~L} /$ day, because of improvements in antiemetics compared with those used before. One important point to note is that timely switching from outpatient chemotherapy to inpatient chemotherapy should be considered for patients with any severe gastrointestinal toxicity or inadequate oral hydration.

In conclusion, outpatient chemotherapy with S-1 + CDDP in advanced gastric cancer patients can be safely and effectively performed in Japan with appropriate patient selection and supportive treatment. However, the long period of infusion (more than $5 \mathrm{~h}$ ) on the day of CDDP administration is a major issue. Further improvement in outpatient chemotherapy units is essential to allow the general use of outpatient chemotherapy with standarddose CDDP in Japan.

Acknowledgments This work was supported by a Grant-in-Aid for Cancer Research [20S-3] from the Ministry of Health, Labor, and Welfare of Japan.

Conflict of interest None declared. 


\section{References}

1. Inoue M, Tsugane S. Epidemiology of gastric cancer in Japan. Postgrad Med J. 2005;81:419-24.

2. Murad AM, Santiago FF, Petroianu A, Rocha PR, Rodrigues MA, Rausch M. Modified therapy with 5-fluorouracil, doxorubicin, and methotrexate in advanced gastric cancer. Cancer. 1993;72:37-41.

3. Ohtsu A, Shimada Y, Shirao K, Boku N, Hyodo I, Saito H, et al. Randomized phase III trial of fluorouracil alone versus fluorouracil plus cisplatin versus uracil and tegafur plus mitomycin in patients with unresectable, advanced gastric cancer: the Japan Clinical Oncology Group Study (JCOG9205). J Clin Oncol. 2003;21:54-9.

4. Boku N, Yamamoto S, Fukuda H, Shirao K, Doi T, Sawaki A, et al. Fluorouracil versus combination of irinotecan plus cisplatin versus S-1 in metastatic gastric cancer: a randomised phase 3 study. Lancet Oncol. 2009;10:1063-9.

5. Koizumi W, Narahara H, Hara T, Takagane A, Akiya T, Takagi M, et al. S-1 plus cisplatin versus S-1 alone for first-line treatment of advanced gastric cancer (SPIRITS trial): a phase III trial. Lancet Oncol. 2008;9:215-21.

6. Jin M, Lu H, Li J, Shen L, Chen Z, Shi Y, et al. Randomized 3-armed phase III study of S-1 monotherapy versus S-1/CDDP (SP) versus 5-FU/CDDP (FP) in patients (pts) with advanced gastric cancer (AGC): SC-101 study. J Clin Oncol. 2008;26 (suppl):abstr 4533.

7. Ajani JA, Rodriguez W, Bodoky G, Moiseyenko V, Lichinitser $\mathrm{M}$, Gorbunova V, et al. Multicenter phase III comparison of cisplatin/S-1 with cisplatin/infusional fluorouracil in advanced gastric or gastroesophageal adenocarcinoma study: the FLAGS trial. J Clin Oncol. 2010;28:1547-53.

8. Ando M, Saka H. Estimated number of cancer patients treated on an outpatient basis in Japan. Gan To Kagaku Ryoho. 2005;32:647-51 (in Japanese).
9. Reed E. Cisplatin and its analogs. In: DeVita VTJ, Lawrence TS, Rosenberg SA, editors. Cancer: principles and practice of oncology. 8th ed. Philadelphia: Lippincott Williams \& Wilkins; 2008. p. 419-26.

10. Tiseo M, Martelli O, Mancuso A, Sormani MP, Bruzzi P, Di Salvia R, et al. Short hydration regimen and nephrotoxicity of intermediate to high-dose cisplatin-based chemotherapy for outpatient treatment in lung cancer and mesothelioma. Tumori. 2007;93:138-44.

11. Gralla RJ, Osoba D, Kris MG, Kirkbride P, Hesketh PJ, Chinnery $\mathrm{LW}$, et al. Recommendations for the use of antiemetics: evidence-based, clinical practice guidelines. American Society of Clinical Oncology. J Clin Oncol. 1999;17:2971-94.

12. Nakashima K, Hironaka S, Boku N, Onozawa Y, Fukutomi A, Yamazaki $\mathrm{K}$, et al. Irinotecan plus cisplatin therapy and S-1 plus cisplatin therapy for advanced or recurrent gastric cancer in a single institution. Jpn J Clin Oncol. 2008;38:810-5.

13. Sasaki Y, Tamura T, Eguchi K, Shinkai T, Fujiwara Y, Fukuda $\mathrm{M}$, et al. Pharmacokinetics of (glycolate- $0,0^{\prime}$ )-diammine platinum (II), a new platinum derivative, in comparison with cisplatin and carboplatin. Cancer Chemother Pharmacol. 1989;23:243-6.

14. Mor V, Stalker MZ, Gralla R, Scher HI, Cimma C, Park D, et al. Day hospital as an alternative to inpatient care for cancer patients: a random assignment trial. J Clin Epidemiol. 1988;41:771-85.

15. Uramoto H, Kagami S, Iwashige A, Tsukada J. Evaluation of the quality of life between inpatients and outpatients receiving cancer chemotherapy in Japan. Anticancer Res. 2007;27:1127-32.

16. Cox KM, Goel S, O’Connell RL, Boyer M, Beale PJ, Simes RJ, et al. A randomised crossover trial comparing inpatient and outpatient administration of high dose cisplatin. Intern Med J. 2011;41:172-8. 